\title{
Effects of Shearing Conditions on Crystalline Orientation and Relaxation in Polyethylene
}

\author{
E. S. HSIUE, R. E. ROBERTSON, ${ }^{*}$ and G. S. Y. YEH \\ Department of Chemical Engineering and Macromolecular Research Center \\ University of Michigan \\ Ann Arbor, Michigan 48109
}

\begin{abstract}
The effects of shearing conditions (i.e., shear temperature and shear rate) on the degree of orientation of polyethylene (Marlex 6006) and to what extent the induced orientation could be relaxed were examined in this study. Two types of samples were prepared: namely, SIC (shear-induced crystallization) and non-SIC samples. The SIC samples show induction times and possess a high degree of $c$-axis orientation along the shear direction. The induced orientation of SIC samples can be relaxed to a small extent but does not reach a steady value. NonSIC samples do not show induction times and they show low degrees of $c$-axis orientation. The induced orientation of nonSIC samples can be relaxed to a steady state value with an activation energy of $90 \mathrm{~kJ} / \mathrm{mole}$. Our results also indicate that, when the shear temperature is at and above $145^{\circ} \mathrm{C}$, polyethylene can be sheared up to $200 \mathrm{X}$ without introducing any significant molecular orientation even at very high shear rates.
\end{abstract}

\section{INTRODUCTION}

$\mathbf{T}$ o increase the transverse mechanical properties of a continuous fiber-reinforced thermoplastic, it is desirable to have an isotropic matrix resin after stamping. However, considerations on the fiber rearrangement during stamping indicate that large extents of shear (up to $200 \mathrm{X}$ ) will be induced in the matrix resin (1). It is the purpose of this study to examine the effects of shearing conditions (e.g., shear temperature, shear rate, shear time, and holding time at the shear temperature) on the degree of orientation and to what extent the induced orientation can be relaxed.

The studies were carried out in a Couette rheometer as described by Krueger and Yeh (2). It was reported that for linear polyethylene sheared in a Couette rheometer, shear induced crystallization (SIC) could occur depending on the shear temperature and shear rate $(2$, 5). The onset of SIC can be detected by an increase of the shear torque. The time corresponding to this torque rise is called an induction time (2). SIC samples were found to possess a high degree of $c$-axis orientation along the shear direction $(2,3)$.

To examine the extent of orientation relaxation, the sheared samples were held at the shear temperature for various times and then quenched in ice water. Hermans' orientation functions (6), calculated from the wide angle X-ray scattering (WAXS) intensities of the quenched samples, were used to quantify the extent of the crystalline orientation relaxation at various shear temperatures and holding times. In the literature there

* Present address; Ford Motor Company, Scientific Research Laboratory, Dearborn, MI 48121. was one report related to relaxation study in the melt. Furukawa, et al. (7) studied annealing polyethylene $\left(\bar{M}_{w}=120,000\right)$, which had been extruded at $170^{\circ} \mathrm{C}$ up to $500 \mathrm{X}$, at temperatures ranging between $135^{\circ} \mathrm{C}$ and $170^{\circ} \mathrm{C}\left(>T_{m}\right)$. They showed that the retraction of samples followed an Arrhenius pattern with an activation energy of $50 \mathrm{~kJ} / \mathrm{mole}$.

\section{EXPERIMENTAL}

Linear polyethylene Marlex $6006\left(\bar{M}_{w}=150,000\right.$ and $\bar{M}_{n}=18,000$ as reported by the manufacturer), obtained from Phillips Petroleum Company, was used in this study. Its melting temperature $T_{m}$ is $132.5^{\circ} \mathrm{C}$ based on the peak position of the differential scanning calorimeter (DSC) endotherm measured at a heating rate of $10^{\circ} \mathrm{C}$ per minute.

The sample was first compression-molded into two semicylinders (1.64 millimeters thick and 61.25 millimeters long). They were then loaded into the cavity between two concentric cylinders of the Couette rheometer with $\mathrm{O}$-rings at both ends. The sample was then melted by placing the rheometer in the oven $\left(180^{\circ} \mathrm{C}\right)$ for about 10 minutes. The oven was adjusted to the desired shear temperature $T_{s}$. After reaching $T_{s}$, the sample melt was sheared at a given shear rate $\dot{\gamma}$ for a shear time $t_{s}$ to reach either $100 \mathrm{X}$ or $200 \mathrm{X}$ shear extent. After shearing, some of the samples were held at $T_{s}$ for various holding times $t_{h}$ before quenching in ice water. The prepared samples were grouped in three different series A, $\mathrm{B}$, and $\mathrm{C}$ as, shown in Fig. 1.

The degree of crystalline orientation of the quenched samples was measured by wide angle X-ray scattering 

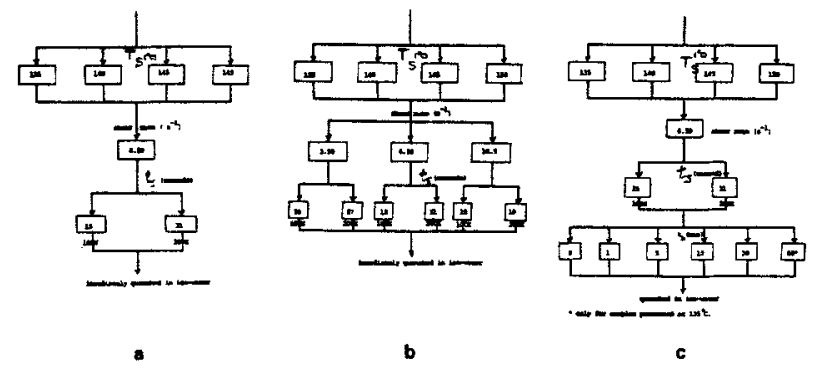

Fig. 1. Sample series A, B, and C. Samples in series A were prepared at $6.5 \mathrm{~s}^{-1}$ shear rate to $100 \mathrm{X}$ or $200 \mathrm{X}$ at different shear temperatures. Samples in series B were prepared at three shear rates $\left(3.5,6.5\right.$ and $\left.10.5 \mathrm{~s}^{-1}\right)$ to $100 \mathrm{X}$ or $200 \mathrm{X}$ at four shear temperatures. Samples in series $C$ were prepared at $6.5 \mathrm{~s}^{-1}$ shear rate to $100 \mathrm{X}$ or $200 \mathrm{X}$ and at four shear temperatures for different holding times.

(WAXS) using Ni-filtered CuK $\alpha$ radiation $(\lambda=0.154$ nanometer) operated at $40 \mathrm{kV}$ and $15 \mathrm{ma}$. A 10 by $10 \mathrm{mil}-$ limeter specimen was cut from the sheared sample and mounted in the sample holder with the shear direction normal to the X-ray incident beam. In order to measure the WAXS intensities at different azimuthal angles, a Norelco pole figure device was used to rotate the specimen azimuthally at a rate of 22.5 degrees per minute during the measurement. The scattering intensities along the azimuthal angles from 0 to 90 degrees of the two scattering peaks $I_{110}$ and $I_{200}$ (at Bragg angles $2 \theta=$ $21.8^{\circ}$ and $\left.24.0^{\circ}\right)$ were detected with a Scintillation counter and recorded at every 4.5 degrees. The intensities were corrected for background scattering.

The orientation function for the $a$ axis is deduced from the scattering of the (200) plane (8). The orientation function for the $b$ axis is then obtained from the Wilchinsky relation (9):

$$
f_{b}=1.444 f_{110}-0.444 f_{200}
$$

The orientation function for the $c$ axis can then be obtained:

$$
f_{c}=-\left(f_{a}+f_{b}\right)
$$

A computer program was developed to calculate the orientation functions for the $a, b$, and $c$ axes. The accuracy of the results computed by this program is in the order of \pm 0.01 . The triangle representation of the state of orientation (originated by Stein (8)) showing $f_{a}$ vs $f_{b}$ was also used in this study to get a clearer picture of the state of orientation of our samples.

\section{RESULTS}

Only samples sheared at $135^{\circ} \mathrm{C}$ (the lowest shear temperature) and at various shear rates $(3.5,6.5$, and 10.5 $\left.\mathrm{s}^{-1}\right)$ to $200 \mathrm{X}$ showed induction times indicating that SIC had occurred $(2,5)$. The induction time $t_{i}$ was determined as the rise of shear torque from the torque $v s$. time plot (Fig. 2). In this study, $t_{i}$ were found to be 38 , 25 , and 15 seconds for samples sheared at $135^{\circ} \mathrm{C}$ corresponding to shear rates of $3.50,6.50$, and $10.5 \mathrm{~s}^{-1}$, respectively. For all the other shearing conditions, no induction time was observed indicating the absence of SIC at shear temperature (Fig. 2). Thus, there were two

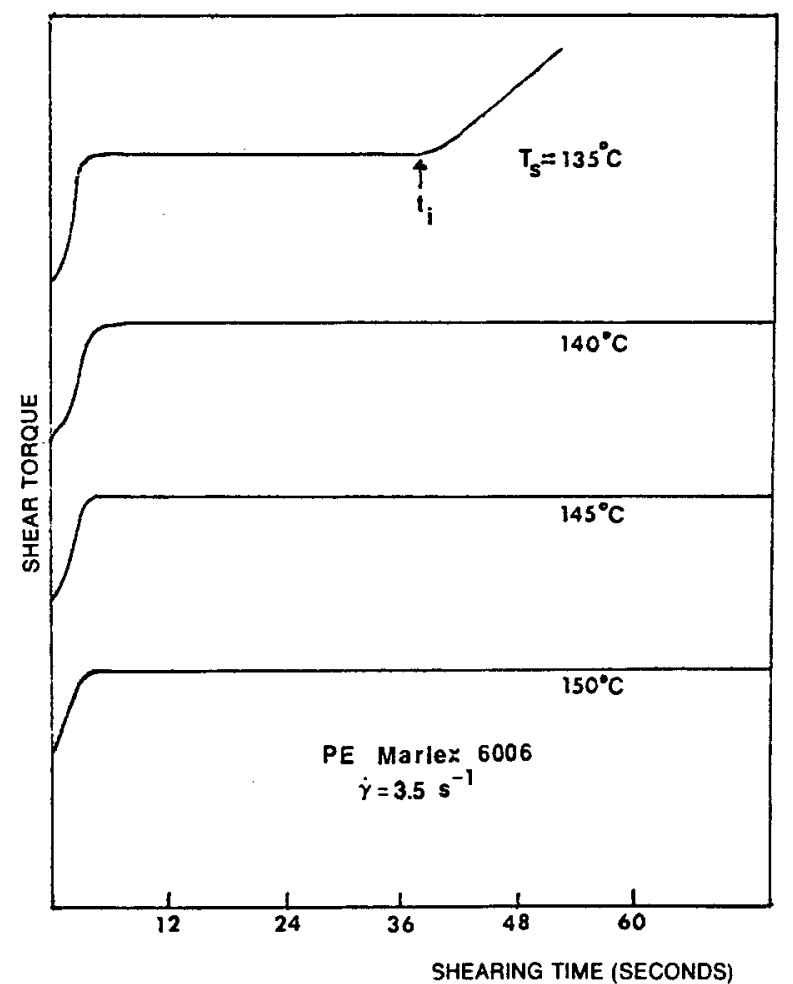

Fig. 2. Examples of shear torque as function of shear time showing the presence (at $\left.135^{\circ} \mathrm{C}\right)$ and absence of induction time $t_{i}$.

types of samples prepared in this study. Samples which showed induction times are designated as SIC samples and those which did not are designated as non-SIC samples.

Three different types of WAXS pattern were obtained (Fig. 3). SIC samples show diffraction maxima near the equator (Fig. $3 a$ ) indicating a high degree of $c$-axis orientation $(2,5)$. Non-SIC samples show either uniform ring patterns (Fig. $3 b$ ) or slightly arced patterns with maxima located about 22.5 degrees from the equator (Fig. 3c). The state of orientation of these three types of WAXS pattern will be examined later in detail.

\section{Processing Effects}

The measured orientation functions of samples belonging to series A (Fig. 1a) are plotted against shear temperature $T_{s}$ (Figs. $4 a$ and $4 b$ ). The SIC sample (sheared at $6.5 \mathrm{~s}^{-1}$ shear rate and at $135^{\circ} \mathrm{C}$ to $200 \mathrm{X}$ ) has $f_{c}$ of about 1 and $f_{a}$ and $f_{b}$ of about -0.4 . For non-SIC samples, $f_{a}$ and $f_{c}$ are positive, $f_{b}$ is negative. In general, the degree of $c$-axis orientation clearly shows a decreasing trend with increasing $T_{s}$.

The measured orientation functions of samples in series B (Fig. $1 b$ ) are plotted vs. shear rates $\dot{\gamma}$ (Fig. 5). For SIC samples, namely, samples processed at $135^{\circ} \mathrm{C}$ at various shear rates to $200 \mathrm{X}, f_{c}$ increases but $f_{a}$ and $f_{b}$ decrease with increasing shear rate (Fig. 5a-i). For nonSIC samples $f_{b}$ decreases and $f_{a}$ and $f_{c}$ increase with increasing shear rate when $T_{s} \leq 140^{\circ} \mathrm{C}$ (Figs. $5 a-i i$ and $5 b-i$ and ii). When $T_{s} \geq 145^{\circ} \mathrm{C}$, the three measured orientation functions are essentially constant for the three shear rates used in this study (Figs. 5a-iii and $i v$ and $5 b-i i i$ and $i v)$. 

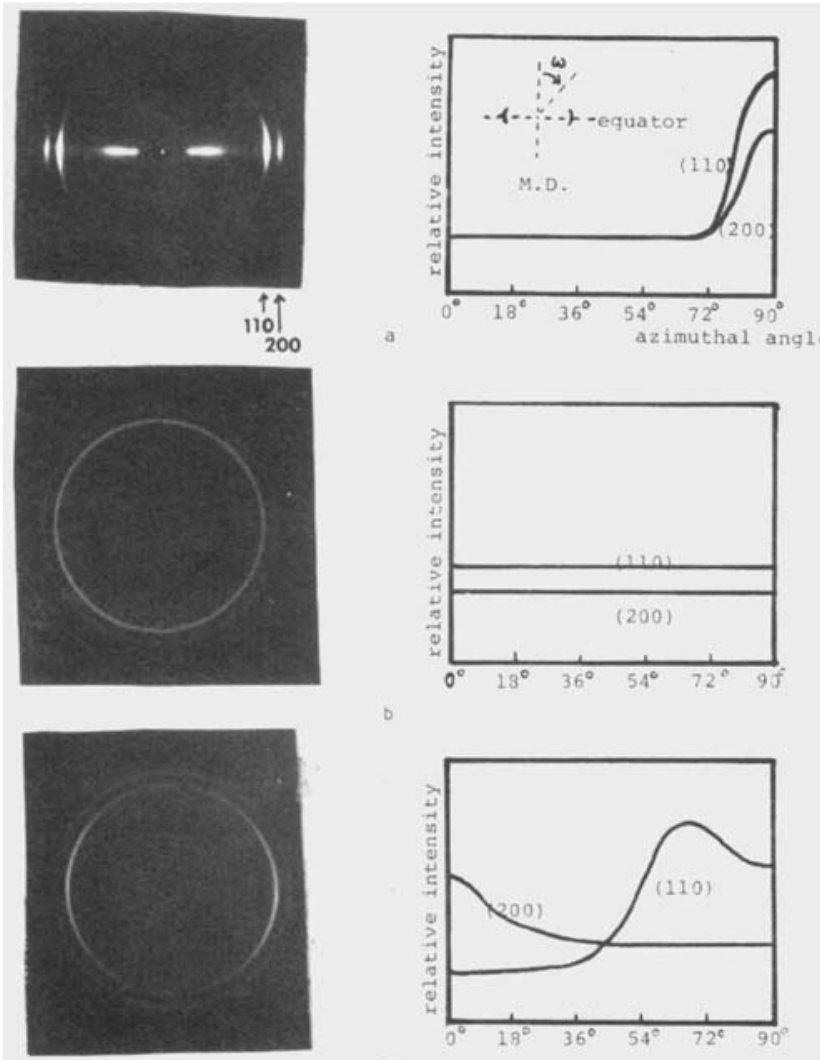

Fig. 3. Examples of WAXS patterns and scattering intensities plotted as function of azimuthal angles $\omega$ defined as shown.

\section{Orientation Relaxation}

The measured orientation functions of samples belonging to series $\mathrm{C}$ (Fig. 1c) are plotted against holding times $t_{h}$ (Fig. 6). The measured orientation functions tend to approach zero (i.e., random orientation) with increasing $t_{h}$ for non-SIC samples, but not for SIC samples (Fig. 6a-i). The induced orientation is almost totally relaxed when $t_{h} \geq 5$ minutes at $t_{s} \geq 145^{\circ} \mathrm{C}$ (Figs. $6 a-i i$ and $i v$ and $6 b-i i$ and $i v)$.

\section{DISCUSSION}

As mentioned earlier, we have essentially prepared two types of samples: SIC and non-SIC samples. SIC samples show induction times (Fig. 2) and have a high
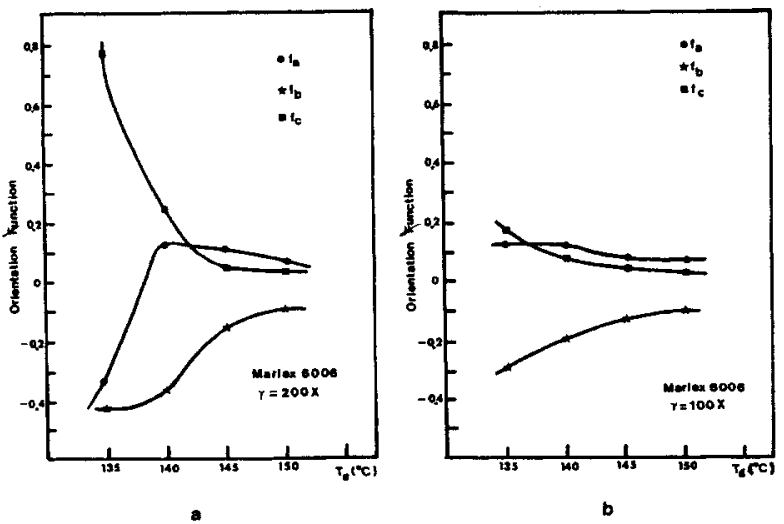

Fig. 4. Orientation functions $f_{a}, f_{b}$, and $f_{c}$ for samples processed at different shear temperature $T_{s}$.
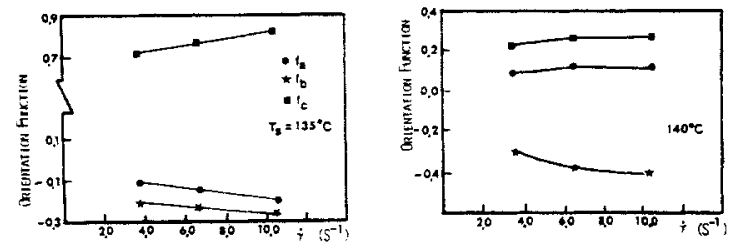

ii

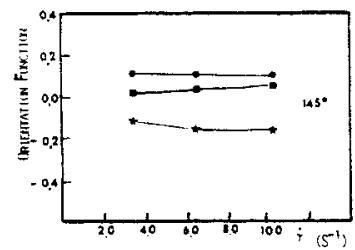

III
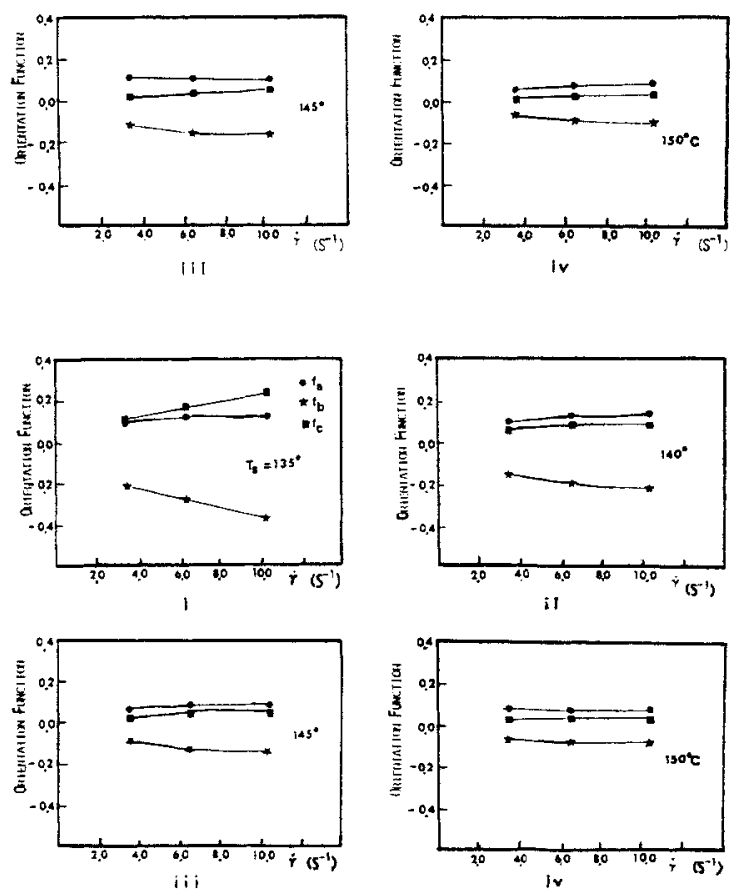

Fig. 5. Orientation functions $f_{a}, f_{b}$, and $f_{c}$ for samples processed at different shear rates. (a) $200 \mathrm{X}$ and (b) 100X.

degree of $c$-axis orientation along the shear direction in agreement with previous findings (2-5). In this study no induction time was observed for non-SIC samples (Fig. 2 ), indicating the absence of SIC in the shearing process. As indicated in the RESULTS section, the measured orientation functions are generally low for nonSIC samples.

In order to get a clearer picture of the state of orientation of our samples, the measured orientation functions for the $a$ and $b$ axes are shown in the triangle plots (Figs. $7 a-e)$. The apex $Z$ represents an orientation with $c$ axis parallel to the shear direction (i.e., $f_{c}=1$ ). The origin $O$ represents a random orientation since $f_{a}, f_{b}$, and $f_{c}$ are equal to zero. The solid (i.e., $f_{b}=-2 f_{a}$ ) and the broken (i.e., $f_{a}=-2 f_{b}$ ) lines represent two different models; namely, Keller's row nucleation model (10) and the $a$-axis orientation model proposed by Holmes and Palmer (11). Both models were developed to explain the state of orientation of samples with typical WAXS patterns like Fig. 3(c). In the row nucleation model (solid line in Fig. 7), the $b$-axis has a tendency to be oriented perpendicular to the deformation direction and the $a$ and $c$ axes are randomly oriented about $b$ axis. In the $a$-axis orientation model (broken line in Fig. 7), the $a$-axis is preferentially oriented parallel to the deformation direction and the $b$ and $c$ axes are randomly oriented about the $a$ axis. 

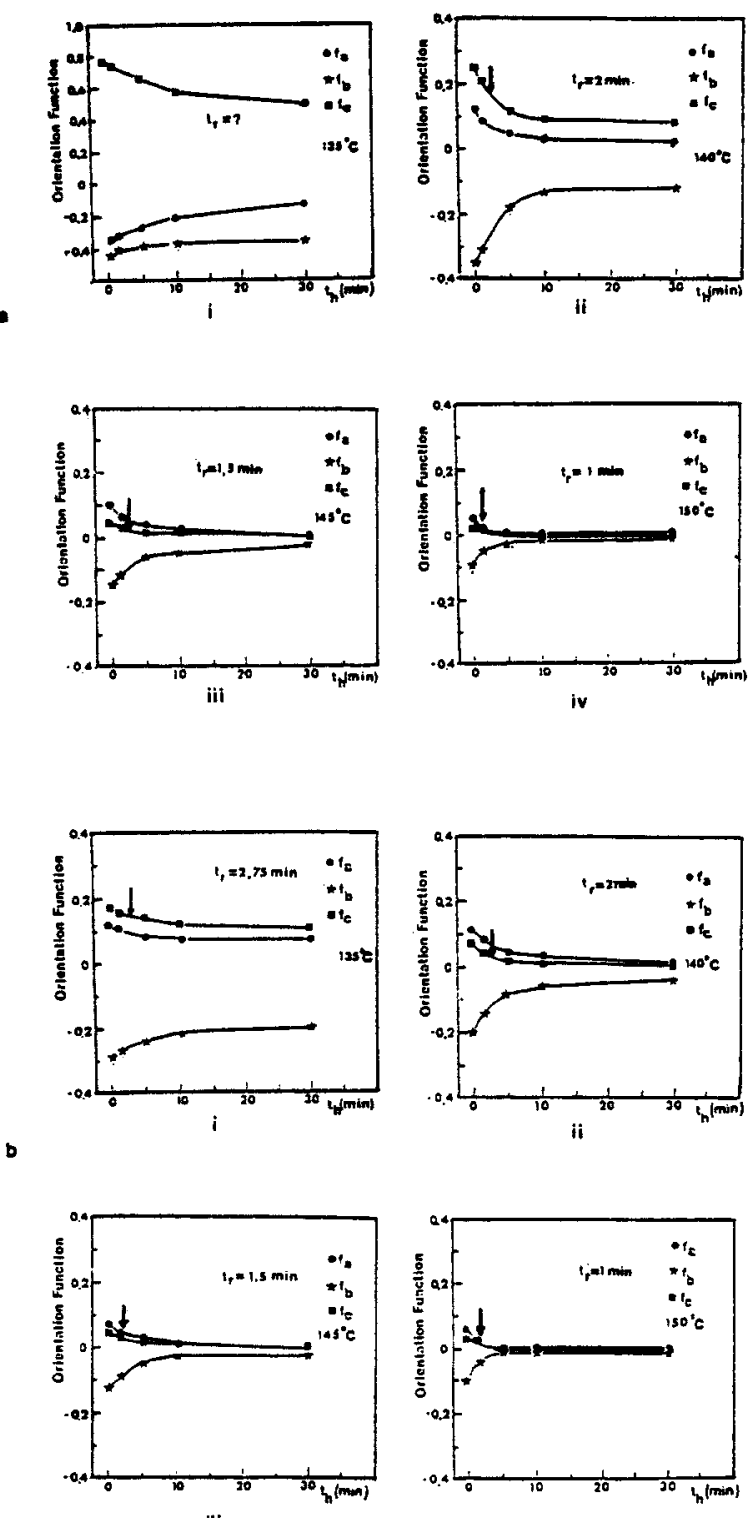

Fig. 6. Orientation functions $f_{a}, f_{b}$, and $f_{c}$ for samples processed at different holding times. (a) $200 \mathrm{X}$ and (b) 100X. Arrow indicates relaxation time $t_{r}$.

The locations of our samples as shown by the dots in Figs. $7 a-e$ indicate that there are clearly three different states of orientation:

I. SIC samples (sheared at $135^{\circ} \mathrm{C}$ to $200 \mathrm{X}$ ) are nearly always located near apex $Z$ (Figs. $7 a-i i, 7 b-i$ and $7 d-i$ ), indicating a high degree of $c$-axis orientation along the shear direction. $f_{a}$ and $f_{b}$ are of about -0.4 , indicating that $a$ and $b$ axes are almost perpendicular to the shear direction.

II. A second group of samples $\left(T_{s} \geq 145^{\circ} \mathrm{C}\right.$ and $t_{h} \geq 5$ minutes) are located near the origin $O$ (Figs. $7 d$-iii and $i v$ and $7 e-i i i$ and $i v)$, indicating essentially a random spherulitic-type morphology (12).

III. The last group covers samples located between I and II (e.g., Fig. 7a-i). Samples in this category have typical WAXS pattern like Fig. $3 c$. Similar WAXS patterns were obtained by crystallizing bulk polyethylene at low elongations (10-11, 13-15). Most of our samples
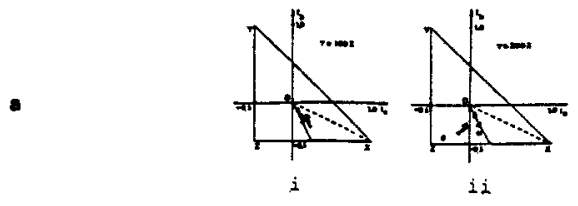
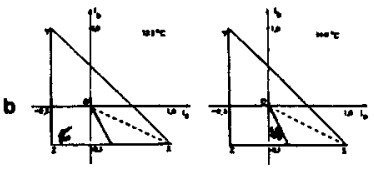

ii
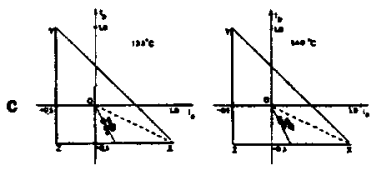

ii
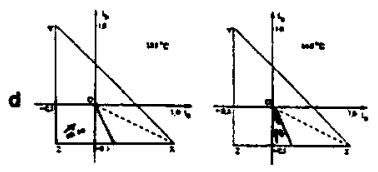

ii
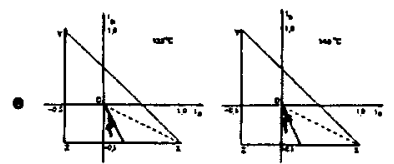

ii

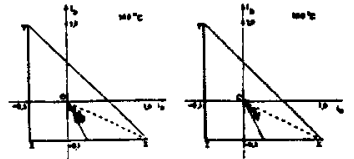

$i: i$

iv

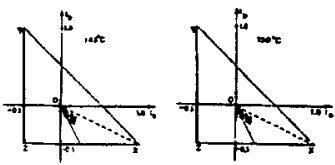

iii

iv

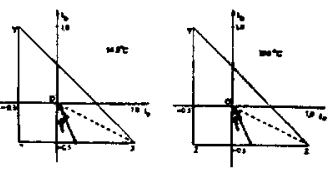

i i i
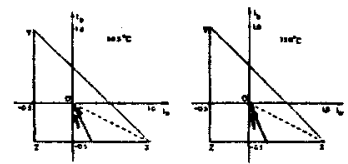

$i i \dot{i}$

$\vdots \mathrm{v}$
Fig. 7. Triangle representation of state of orientation. (a) samples processed at different shear temperature $T_{s},(b)$ at different shear rate $\dot{\gamma}$ for a shear of $200 \mathrm{X},(c)$ at different shear rate $\dot{\gamma}$ for a shear of $100 \mathrm{X},(d)$ at different holding time $t_{h}$ for a shear of $200 \mathrm{X}$ and (e) at different holding time $t_{h}$ for a shear of $100 \mathrm{X}$. Arrow in each triangle indicates increase in processing parame$\operatorname{ter} T_{8}, \dot{\gamma}$, or $t_{h}$.

lie closer to the solid line. It is of interest to note that, according to the so-called "row nucleation" model, SIC crystals which form at the shear temperature are depicted to serve as line nuclei for the rows of nearly perpendicular-oriented lamellae which form during quenching (16). However, our results clearly showed that the so called row-nucleated orientation can also be present in our non-SIC samples. This indicates that the presence of the perpendicular-oriented lamellae need not arise from SIC nuclei but could be from nuclei obtained during quenching.

In summary, Stein's triangle plots also show that there are two distinct types of samples: (a) SIC samples (which show induction times) that have a high degree of $c$-axis orientation and (b) non-SIC samples (which do not show induction times) that have a random orientation and/or a "row-nucleated" type orientation.

\section{Processing Effects}

We now consider in more detail the effects of the shearing conditions on the degree of orientation. Our results show that the degree of orientation decreases with increasing $T_{s}$ (Fig. 4). For samples processed at $T_{s}$ 
$\geq 145^{\circ} \mathrm{C}$, little orientation is observed even when sheared up to $200 \mathrm{X}$. The shear rate has negligible effects on the degree of orientation for samples processed at $T_{s} \geq 145^{\circ} \mathrm{C}$. The measured $f_{c}$ increases less than 0.05 when the shear rate is increased from 3.5 to $10.5 \mathrm{~s}^{-1}$ (Figs. 5a-iii and $i v$ and $5 b-i i i$ and $i v$ ).

As mentioned in the INTRODUCTION, it is desirable to have an isotropic matrix after stamping to increase the transverse mechanical properties of the thermoplastic composite. Under the specific conditions used in this study, polyethylene Malex 6006 can be sheared up to $200 \mathrm{X}$ without introducing any significant orientation as long as the shear temperature is at and above $145^{\circ} \mathrm{C}$. At such high $T_{s}$ (lack of SIC) the shear rate has negligible effect on the degree of orientation. Therefore, a shorter cycle time, which is economically preferred, can be achieved by using high shear rates to reach the desired extents of shear.

\section{Orientation Relaxation}

From the results on the effects of holding times on the degree of orientation (Fig. 6), the kinetics of the orientation relaxation can be studied by examining the relaxation of $f_{c} v s$. $t_{h}$. A relaxation time $t_{r}$ was characterized as the time at which $f_{c}$ was equal to one-half of the sum of the starting and the final $f_{c}$ values. For instance, the initial $f_{c}$ is 0.03 and the final $f_{c}$ is 0.01 in Fig. $6 b$-iv. The average $f_{c}$, therefore, is 0.02 with a corresponding relaxation time $t_{r}$ of 1 minute.

No $t_{r}$ can be assigned for SIC sample (Fig. 6a-i) since $f_{c}$ does not reach a steady value even after long $t_{h}$ up to 60 minutes. This can be attributed to the presence of SIC crystals. Since these SIC crystals will have a higher melting temperature than the temperature at which they were sheared $\left(135^{\circ} \mathrm{C}\right)$, they should remain stable at $135^{\circ} \mathrm{C}$ and consequently maintain some of their orientations. It is of interest to note that for the non-SIC samples, no difference in $t_{r}$ is observed between samples sheared for different extents (200X (Figs. $6 a-i i$ to $i v$ ) and $100 \mathrm{X}$ (Figs. $6 b$-ii to $i v)$ ). At the present time, we have no explanation for this observation.

A plot of $\ln \left(1 / t_{r}\right) v s .1 / T_{s}$ is shown in Fig. 8. The relaxation is found to follow the Arrhenius plot with $E_{a}=90$ $\mathrm{kJ} / \mathrm{mole}$. The value of $E_{a}$ is comparable to $E_{a}=50$ $\mathrm{kJ} /$ mole reported by Furukawa, et al. (7), for the retraction in the melt of melt-spun polyethylene samples. The difference in $E_{a}$ could be attributed to the fact that, in this study, the $E_{a}$ was obtained from the crystalline orientation relaxation, whereas Furukawa, et al. (7), determined $E_{a}$ from the retraction measurements which arise primarily from the amorphous chain segments. In order to examine more fully these various relaxation effects, measurements on the relaxation of amorphous orientation should be carried out.

\section{CONCLUSION}

Two types of samples were prepared in this study: namely, SIC and non-SIC samples. The SIC samples show induction times and possess a high degree of $c$-axis orientation along the shear direction confirming previous findings (2-5). Non-SIC samples do not show in-

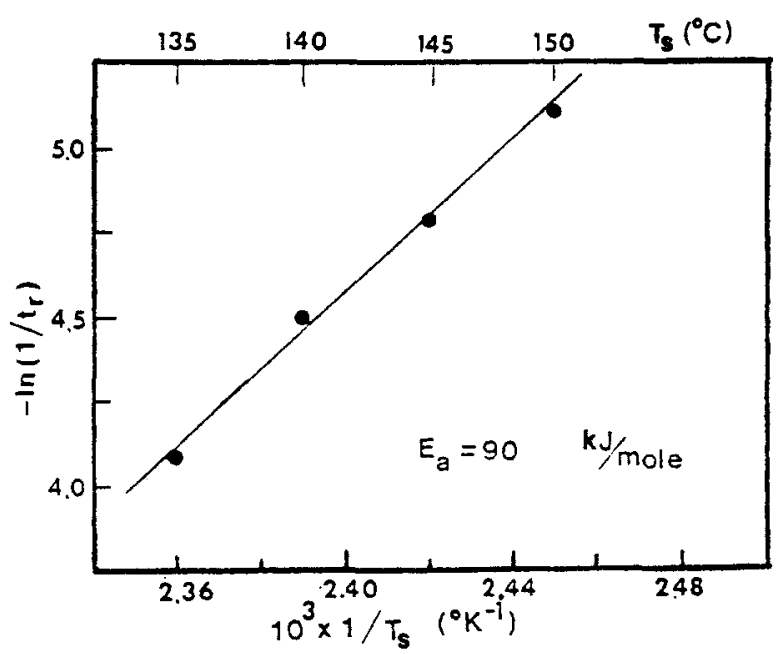

Fig. 8. Arrhenius plot for the orientation relaxation time.

duction times and they generally have either a random orientation or an orientation similar to the row nucleation model (11). However, our non-SIC samples do not have SIC crystals and still possess a row-nucleated orientation.

Under our specific experimental conditions, it is possible to shear polyethylene Marlex 6006 up to $200 \mathrm{X}$ without introducing any significant orientation as long as $T_{s} \geq 145^{\circ} \mathrm{C}$. At such high shear temperatures, the shear rate has negligible effects on the degree of orientation.

The shear-induced crystalline orientation can be relaxed as long as the SIC crystals are not formed. For Marlex 6006 non-SIC samples, the orientation relaxation can be described by an Arrhenius equation with $E_{a}$ of $90 \mathrm{~kJ} / \mathrm{mole}$.

\section{ACKNOWLEDGMENT}

This research was supported in part by US Department of Energy (under contract No. ER-78-C-02-5008) and in part by the Army Research Office of Durham (DAAG-29-81-C-0026).

\section{REFERENCES}

1. R. E. Robertson, E. S. Hsiue, E. N. Sickafus, and G. S. Y. Yeh, Polym. Compos, 2, 126 (1981).

2. D. L. Krueger and G. S. Y. Yeh, J. Appl. Phys., 43, 4739 (1972).

3. G. S. Y. Yeh, Polym. Eng. Sci., 16, 138 (1976).

4. G. S. Y. Yeh, Polym. Eng. Sci., 16, 145 (1976).

5. G. S. Y. Yeh, K. Z. Hong, and D. L. Krueger, Polym. Eng. Sci., 19, 401 (1979).

6. P. H. Hermans and P. Platzek, Kolloid., 88, 68 (1939).

7. J. Furukawa, T. Kiato, S. Yamashita, and S. Ohya, J. Polym. Sci., Part A-1, 9, 299 (1971).

8. R. S. Stein, J. Polym. Sci., 31, 327 (1958).

9. Z. W. Wilchinsky, J. Appl. Phys., 30, 792 (1959).

10. A. Keller, J. Polym. Sci., XV, 31 (1955).

11. D. R. Holmer and R. P. Palmer, J. Polym. Sci, 31, 345(1958).

12. P. H. Geil, "Polymer Single Crystals," Interscience Div., John-Wiley, New York (1968).

13. A. Brown, J. Appl. Phys., 20, 552 (1949).

14. R. A. Horsley and H. A. Nancarrow, Brit. J. Appl. Phys., 2, 345 (1951).

15. P. R. Holmes, R. P. Palmer, and C. W. Bunn, Nature, 171, 1104 (1953).

16. A. Keller and M. J. Machin, J. Macromol. Sci., B1, 41 (1967). 